energetic state of activation in the body. It is the physical intensity level. The level of stress or activation can vary from deep sleep to intense excitement. The idea of peak activation level is that for every skill there is an optimal level of activation, a level that corresponds to a maximum performance level. The peak stress state is the level of energy and intensity that relates to an athlete's best performance level. Top achievers in sports operate in or near to their peak stress level in pressure situations. In the present research paper the Indian male wrestlers were studied on select psycho-physiological variables and their profiles were analysed. The wrestlers were then exposed to the Progressive Muscle Relaxation training for 45 days and their responses were recorded on the four psycho-physiological variables. Thus the complete pretesting and the post training profiling was done and the outcomes were interpreted. The analysis of the present scenario of the psychological condition of the Indian male wrestlers were done and accordingly the recommendations are being made for the improvement of the performance of Indian wrestlers so that they can perform better by overcoming the psychological hurdles at the elite level.

\title{
188 PSYCHO PHYSIOLOGICAL PROFILES OF INDIAN WRESTLERS
}

Vikram Singh, ${ }^{1}$ Sanjeev P Sahni ${ }^{2}$ Department of Physical Education, Jawaharlal Nehru University, New Delhi, India; ${ }^{2}$ Education Initiatives, Jindal Group of Companies, New Delhi, India

\subsection{6/bjsm.2010.078725.188}

At the elite level the mental or psychological factors affect the sportsperson's abilities. For developing greater pressure-handling ability, we need to examine individuals who are exposed to handling risk and pressure. Stress refers to the physical, 